\title{
Research on the Synthesis of Multi-Frequency Waveform and High Precision Synchronization Technology in Frequency Domain Electromagnetic Transmitter
}

\author{
Bo Wang, Ming Deng, Meng Wang, Zheng Xiang, Shuxiang Wang \\ Key Laboratory of Geo-detection, Ministry of Education, \\ China University of Geosciences (Beijing), Beijing, China
}

\begin{abstract}
As a critical component in electromagnetic exploration device, there are many issues in frequency domain electromagnetic transmitter to be addressed in the aspect of cover range of frequency points, operation efficiency under wild condition and high precision synchronization transmission as well as other aspects. The application of direct digital frequency synthesize technology (DDS) and the design of complex programmable logic device (CPLD) allow us to adjust the transmitting signal frequency of transmitter to any frequency within the range of $10 \mathrm{kHz}-0.01 \mathrm{~Hz}$; with the powerful logic operation capability of CPLD, a simple, reliable multi-frequency power supply wave shape with balanced power distribution is added in the basis of common frequency sweeping function, which significantly improved the operation efficiency of field electromagnetic prospecting; the design is equipped with constant temperature crystal oscillator calibration circuit based on GPS, consequently realize the high precision synchronization transmitting and the technological support for the synchronic signal collection. It is approved in a series of sink experiments and wild experiments that the transmitting wave shape synthesized by the transmitter could fully satisfy the requirement of wild electromagnetic exploration, the frequency point is high in precision, with stable frequency and balanced multi-frequency power distribution, the transmitting synchronization error is in nanosecond class, the arrangement of dead time is rational.
\end{abstract}

Keywords - frequency domain electromagnetic transmitter; DDS; CPLD; multi-frequency waveform; high precision synchronization transmission

\section{INTRODUCTION}

Frequency domain electromagnetic method is the method to research on the ground steady-state response to the frequency change, this method has been widely used in metal mineral exploration, coal mine investigation, environment hydrological survey as well as other fields ${ }^{[1]}$. At present, the representative frequency domain electromagnetic transmitter are GDP-32 from American company Zonge and V8 from Canadian company Phoenix etc ${ }^{[2]}$. In China, different research institutes contributed to the development in this case such as ATTEM-II from University of Jilin, WDC-2 from Langfang geophysical exploration institute, WTEM-1 from Chongqing University, wide field electromagnetic transmitter developed by Central South University ${ }^{[3-6]}$. The transmitting frequency point of most transmitter at present is realized by frequency division for fixed frequency point, which could not support the transmitting of random frequency, and limit the general application of transmitter ${ }^{[7]}$; the common frequency sweeping method is used in wild exploration, i.e. transmit the wave shape of certain one frequency to the underground at one time, however the efficiency is low; although GPS Global Positioning System and high precision constant temperature crystal oscillator were applied in some transmitting systems to realize synchronized transmission, it is hard to preserve long time synchronized transmission as the issue of frequency drift in constant temperature crystal oscillator was not addressed ${ }^{[8]}$. All these issues need serious attention in the development of frequency domain electromagnetic transmitter.

Regarding the above issues, the synthesize of transmitting wave shape of random frequency is realized based on the advantage of DDS in digital frequency synthesize and the proper dead time from CPLD; a simple and reliable multi-frequency transmitting wave shape is designed in this research, which is approved by Fourier transform operation that the power distributed in each frequency point is balanced; the issue of time drift in constant temperature crystal oscillator is solved by the measurement of time difference between the GPS pulse per second signal and the $1 \mathrm{~Hz}$ frequency division signal from constant temperature crystal oscillator, and regulate the control voltage range of crystal oscillator with the digital to analog converter with the purpose to calibrate the crystal oscillator, high precision synchronized transmitting is realized and the relevant functions have been testified in wild experiment.

\section{SyNTHESIS OF MULTI-FREQUENCY CURRENT SUPPLY WAVE}

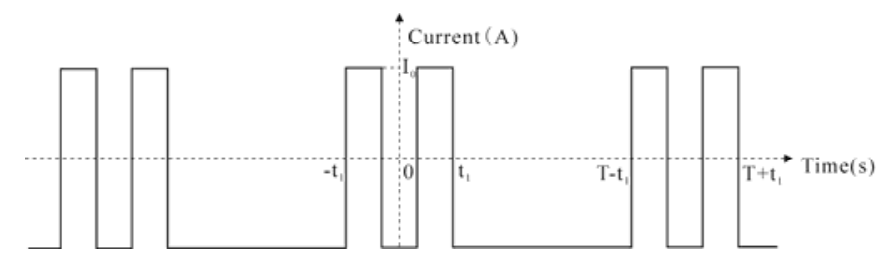

Fig. 1 Multi-frequency current supply wave 
The multi-frequency current supply wave in Fig.1 is designed for the research, taking $t_{1}=\frac{3}{1600} s, T=\frac{1}{100} s$ as example, the expression in Fig. 1 is

$f(t)=2 I_{0}\left(G_{3 / 800}(t)-G_{1 / 800}(t)\right) * \delta_{1 / 100}(t)$

where $G_{T}(t)$ represents the rectangular pulse between $-T / 2$ and $T / 2, \delta_{T}(t)$ represents the cycle unit impulse sequence. The Fourier transform of $f(t)$ is

$F(w)=2 I_{0}\left(\frac{3 \pi}{4} S a\left(\frac{3 w}{1600}\right)-\frac{\pi}{4} S a\left(\frac{w}{1600}\right)\right) \cdot \sum_{n=-\infty}^{\infty} \delta(w-200 n \pi)$

according to the above expression, when $w=200 n \pi$ (i.e. $f=100 n), F(w)$ has value, the power is mainly distributed in four frequency points as $100 \mathrm{~Hz}, 300 \mathrm{~Hz}, 400 \mathrm{~Hz}$ and $500 \mathrm{~Hz}$, the proportion of the amplitude value of each frequency point in that of fundamental wave is $69 \%, 55 \%, 64 \%$ and $33 \%$, the power distribution is balanced, the wave in other frequency point is composed of harmonic wave.

\section{High PRECISION TIME SyNCHRONIZATION TRANSMISSION}

The introduction of constant temperature crystal oscillator calibration technology based on GPS signal would facilitate the realization of high precision time synchronization in frequency domain electrical prospecting. At first, CPLD would divide the frequency of constant temperature crystal oscillator by $1 \mathrm{~Hz}$, the internal value between measured $1 \mathrm{~Hz}$ signal and GPS second pulse signal would be delivered to micro-processor ARM, then ARM could calculate the numerical relationship between control voltage range of crystal oscillator and the frequency drift, regulate the control voltage range of crystal oscillator with the digital to analog converter with the purpose to calibrate the crystal oscillator, then use the fixed time signal PPS generated from crystal oscillator frequency division as synchronize signal to realize high precision synchronized transmission. As the issue of crystal oscillator frequency drift with time has been addressed, we obtained desired synchronization signal and ensured the precision and stability of the transmission frequency of the transmitter. Fig. 2 is the schematic diagram of crystal oscillator frequency calibration system.

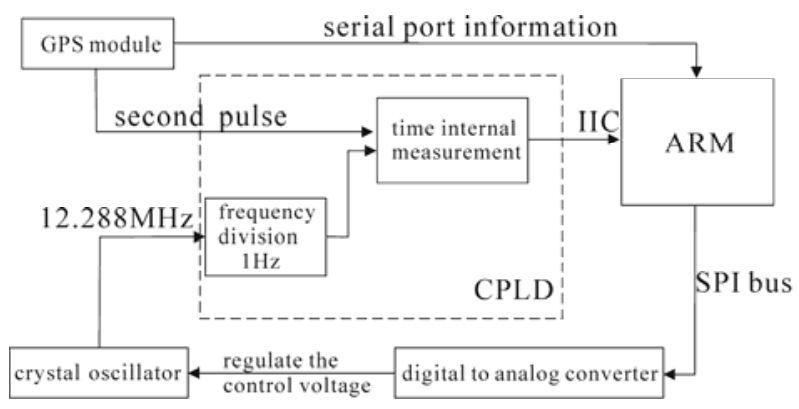

Fig. 2 Schematic diagram of crystal oscillator frequency calibration

\section{EXPERIMENT TEST}

Fig. 3 is the spectrogram of dual frequency current supply wave shape synthesized by $100 \mathrm{~Hz}$ and $400 \mathrm{~Hz}$ control signal, which is consistent with the calculation result, the power is mainly concentrated in four frequency points as $100 \mathrm{~Hz}, 300 \mathrm{~Hz}, 400 \mathrm{~Hz}$ and $500 \mathrm{~Hz}$. The diagram showed that the power in multifrequency wav shape is balanced, the precision of frequency point is high, and satisfies the planed design effect.

Fig. 4 is the measurement diagram of time different between the synchronize signal PPS from crystal oscillator frequency division and GPS second pulse when crystal oscillator calibration technology was not introduced. Fig. 4 showed that the synchronization signal shifted 48us in total under continuous operation for 5 hours which is much better than the precision requirement in electromagnetic exploration. In order to test the synchronization precision of this system, $120 \mathrm{~Hz}$ single frequency signal has been transmitted continuously for 5 hours, one synchronization error is recorded for every $10 \mathrm{~min}$, and the record result is as in Fig. 5. We can see from the comparison between Fig. 4 and Fig. 5 that the precision of system synchronization error is better than 111ns under long time continuous operation condition, which fully satisfies the requirement on synchronization precision for frequency domain electromagnetic exploration.

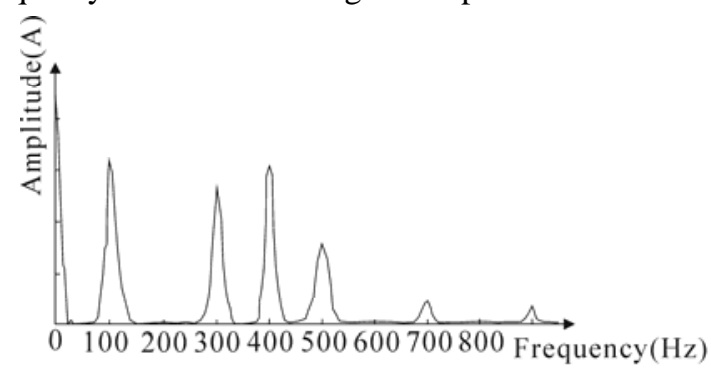

Fig. 3 Spectrogram of multi-frequency current supply wave

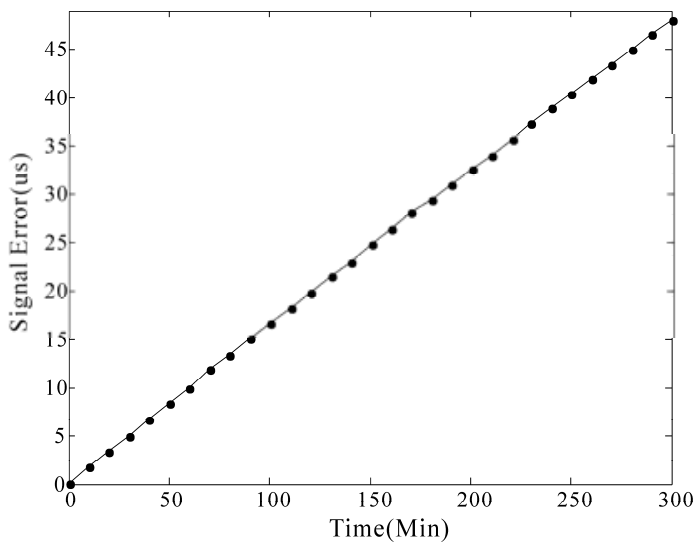

Fig. 4 Synchronization signal error before calibration 


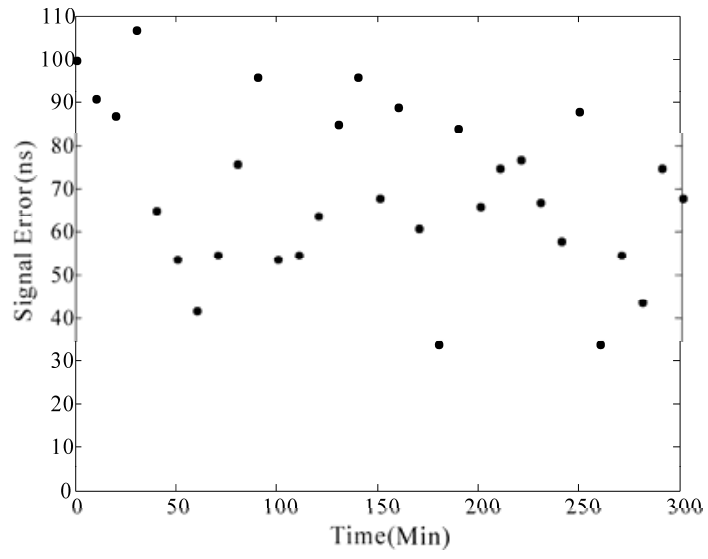

Fig. 5 Synchronization signal error after calibration

\section{CONCLUSIONS}

following conclusions are drawn from research on the synthesis of multi-frequency signal and high precision synchronization technology in frequency domain electromagnetic transmitter: single frequency bipolar rectangular synthesize is realized with the application of DDS and CPLD technologies, the flexibility of control system in the transmitter would be improved under the condition that the precision and stability of transmitting frequency point; a simple and reliable multiple wave shape synthesize plan is proposed to improve the operation efficiency of transmitter in wild condition. The frequency drift issue of constant temperature crystal oscillator is addressed with the application of constant crystal oscillator technology based on GPS and realized the high precision time synchronization of transmitter.

\section{ACKNOWLEDGMENT}

I would like to acknowledge the project in national 863 plan high power borehole-ground electromagnetic imaging system (2014AA06A603), and the fund support from National Science Foundation (41504138) and other organizations, as well as the helpful comment on the literature from my tutor Professor Deng Ming and the assistance from the team.

\section{REFERENCES}

[1] J. H. He, "The new development of frequency domain electro-prospecting," Progress in Geophysics, vol. 22, no. 4, pp. 1250-1254, 2007.

[2] H. T. Zhao, L. H. Liu, K. Wu, J. G. Zhang, and Y. G. Fang, "Voltage stabilized clamping and fast turn-off transient electromagnetic transmitting system," Chinese Journal of Scientific Instrument, vol. 34. no. 4, pp. 803-808, 2013.

[3] S. B. Yu, S. H. Jia, G. Li, and J. L. He, "Implementation of high power transmitting system for grounded source time-domain electromagnetic method,” Foreign Electronic Measurement Technology, vol. 33, no. 7, pp. 49-52, 2014.

[4] F. S. Shi, "A study on high-power multi-function transmitting system," Progress in Geophysics, vol. 24, no. 3, pp. 1109-1114, 2009.

[5] Z. H. Fu, J. L. Zhao, L. W. Zhou, Q. Luo, and X. F. Su, "WTEM fast turn-off transient electromagnetic detection system," Chinese Journal of Scientific Instrument, vol. 29, no. 5, pp. 933-936, 2008.

[6] J. T. Tang, J. Z. Gong, and W. B. Luo, "Invert-Repeated m-sequence pseudorandom signal generator based on CPLD," Chinese Journal of Engineering Geophysics, vol. 5, no. 2, pp. 141-147, 2008.

[7] J. Q. Li, Z. H. Fu, and P. F. Xie, "A complex multi-frequency synthesis technology applied electromagnetic detecting source,” Electrical Measurement and Instrumentation, vol. 46, no. 518, pp. 63-66, 2009.

[8] G. Q. Zuo, W. B. Luo, F. Li, and Y. C. Bai, "Time synchronization design of transmitter and receiver based on GPS,” Geophysical and Geochemical Exploration, vol. 30, no. 2, pp. 158-161, 2006. 